\title{
Intervención educativa en conocimientos, actitudes y prácticas sobre multimicronutrientes, en niños de Chilca, Huancayo - 2017
}

Educational intervention in knowledge, attitudes and practices on multimicronutrients, in children of Chilca, Huancayo - 2017

Diana Esmeralda Andamayo Flores 1,a, Javier Eduardo Curo Yllaconza 1,b, Reneé Valle Elescano ${ }^{1, b}$, Diana Esmeralda Castillo Andamayo ${ }^{1, a}$, Edith Suasnabar Cueva ${ }^{1, c}$, José Efren Vera Cuadros ${ }^{1, b}$, Deisy Miluska Tueros Victorio ${ }^{1, \mathrm{c}}$

\section{RESUMEN}

Objetivos: Mejorar los conocimientos, actitudes y prácticas del consumo de multimicronutrientes en niños menores de 3 años, de la Micro Red de Salud Chilca, mediante una intervención educativa. Material y Métodos: Se utilizó el método científico, siendo una investigación de tipo aplicada, diseño epidemiológico, nivel aplicativo; con una muestra de 65 madres o cuidadoras de niños menores de 5 años en la Micro Red de Salud Chilca, de la provincia de Huancayo. Se realizó una intervención educativa con una fase diagnóstica y una prueba final, para medir los cambios esperados a partir de la intervención. La hipótesis fue contrastada a través de la prueba $\mathrm{t}$ de Student (nivel de significancia 0,05\%). Resultados: Se encontró que los cambios más significativos con relación a los conocimientos sobre el consumo de micronutrientes, se evidenció en lo relacionado a la información que manejan las madres sobre los principales problemas que tiene un niño cuando presenta anemia (incremento de 30,7\%); con relación a las actitudes sobre el consumo de micronutrientes, se logró tener una actitud favorable de las madres, respecto a lo bueno que resulta para el niño consumir el suplemento (incremento de 30,8\%); las prácticas sobre el consumo de micronutrientes en los niños mejoró con relación al consumo del suplemento el día anterior a la visita domiciliaria, logrando un incremento de 30,6\%; además de otros cambios positivos logrados por la intervención educativa. Conclusión: Los conocimientos, actitudes y prácticas sobre el consumo de multimicronutrientes, cambiaron positivamente después de la intervención educativa ( $\mathrm{p}$-valor=0,000).

PALABRAS CLAVE: Anemia, hierro, suplemento, intervención educativa.

\section{SUMMARY:}

Objectives: To improve knowledge, attitudes and practices of multimicronutrient consumption in children under 3 years of age, from the Micro Health Network of Chilca, through an educational intervention. Material and Methods: The scientific method was used, being a research of applied type, epidemiological design, application level; with a sample of 65 mothers or caregivers of children under 5 years of age in the Micro Health Network of Chilca, in the province of Huancayo. An educational intervention was carried out with a diagnostic phase and a final test, to measure the expected changes from the intervention. The hypothesis was contrasted through the 
Student's t test (level of significance $0.05 \%$ ). Results: It was found that the most significant changes in relation to knowledge about micronutrient consumption were evidenced in the information that mothers have about the main problems a child has when they have anemia (increase of 30.7\%); in relation to the attitudes on the micronutrient consumption, it was possible to have a favorable attitude of the mothers, regarding the good thing that results for the child to consume the supplement (increase of 30.8\%); the practices on the consumption of micronutrients in children improved in relation to the consumption of the supplement the day before the home visit, achieving an increase of $30.6 \%$; in addition to other positive changes achieved by the educational intervention. Conclusions: The knowledge, attitudes and practices on the consumption of multimicronutrients, changed positively after the educational intervention $(\mathrm{p}$-value $=0.000)$.

KEYWORDS: Anemia, iron, supplement, educational intervention.

\section{INTRODUCCIÓN}

Según la Organización Mundial de la Salud (1), la anemia afecta en todo el mundo a 1620 millones de personas, por lo que representa uno de los problemas más serios en salud infantil, presentándose la máxima prevalencia en los niños preescolares $(47,4 \%)$; en el Perú la anemia se constituye en la actualidad en un problema de salud pública, según los datos de la Encuesta Demográfica de Salud Familiar 2016, el $33,3 \%$ de niñas y niños menores de cinco años de edad presenta esta deficiencia nutricional; el problema se agrava, si se revisan las cifras según la edad, ya que la anemia afecta principalmente a las niñas y niños menores de 18 meses de edad: $62,1 \%$ en los de 6 a 8 meses de edad, $59,3 \%$ de 12 a 17 meses de edad y $56,7 \%$ de 9 a 11 meses de edad. En los infantes de 18 a 23 meses de edad fue 43,6\%. Mientras que, en los grupos de mayor edad, fueron menores: $29,3 \%$ entre 24 y 35 meses de edad, $24,4 \%$ de 36 a 47 meses de edad y $17,5 \%$ de 48 a 59 meses de edad. Pero un tema realmente alarmante es que, si se revisan los datos en la región Junín, el $41,9 \%$ de los niños de esta zona padecen de este mal (2).

Al confrontar estas cifras con la teoría existente sobre el tema, investigadores como Pollitt, afirman que una simple descripción del crecimiento del cerebro ayuda a comprender el significado que puede tener la deficiencia de hierro como un co-factor necesario o componente estructural de enzimas y moléculas requeridas para el desarrollo y la función exitosa del sistema nervioso, incluyendo la conducción del impulso nervioso (3); es así que la anemia durante los primeros años de vida puede dejar una secuela funcional caracterizada principalmente por un rendimiento escolar por debajo del nivel de los niños no anémicos, presentando principalmente problemas de memoria, de atención y de comprensión; por lo que se puede inferir que, mientras tengamos niños anémicos en el Perú es imposible pensar en una educación con calidad, que nos garantice en el futuro, ciudadanos competitivos en todos los niveles.

Por estas razones existe una creciente preocupación por estudiar el tema anemia y todo lo que implica además de sus factores asociados y la forma como erradicarla; estudios como el realizado por Christensen abordan el tema de adherencia a los suplementos de hierro y concluyen que el poco consumo por parte del niño se debe a intolerancia digestiva y el olvido de las madres para cumplir la dosis (4). Aguirre fue enfático en indicar que en algunos lugares la adherencia del suplemento es menor en los niños con anemia (5). Por su parte Vizuet al final de un estudio demostró que la prevalencia de anemia disminuye a partir del consumo de suplementos (6); e incluso hay estudios que inciden en indicar que los suplementos de hierro son una alternativa efectiva para prevenir y controlar la anemia infantil $(7,8,9)$; Durand añade a este tema la importancia de los conocimientos que tienen las madres acerca de la alimentación y el estado nutricional del niño (10).

Basado en toda la información existente a la fecha, gobiernos como el de Perú, afrontan el problema en los últimos años, a través de la suplementación con hierro mediante el uso de multimicronutrientes (11), pero los resultados alcanzados hasta la fecha fueron negativos, porque en los últimos 5 años la anemia infantil se incrementó en 2,6 puntos porcentuales, lo que significa que actualmente 948000 niños menores de cinco años tienen anemia; a pesar de esto el gobierno actual a través de la Resolución Ministerial N ${ }^{\circ}$ 250-2017/ MINSA- Norma Técnica de Salud, plantea seguir utilizando este tratamiento como la estrategia para disminuir el porcentaje de niños anémicos (11). Sin embargo, estudios como el de Munares demuestran 
que existe una baja prevalencia de adherencia para el consumo de los multimicronutrientes, lo que vuelve crítica la situación y explica posiblemente el por qué actualmente en nuestro País, sigue en aumento los índices de anemia infantil (12).

A partir de lo expuesto, es necesario investigar sobre los problemas relacionados al consumo de estos suplementos (micronutrientes) para los niños, que no logran resolver los problemas de deficiencia de hierro y altas prevalencias de anemia, que, en la mayoría de los casos según los estudios, se relaciona a la no adherencia al tratamiento. Es así que el propósito del presente proyecto de investigación se vuelve relevante desde el punto de vista del impacto en el futuro del Perú. La inversión en nutrición es a corto y a largo plazo una dedicación que va a dar resultados muy satisfactorios; según los avances científicos, a la fecha para cuando un niño llega a la edad escolar, alrededor del $80 \%$ de su cerebro ya se ha desarrollado, lo que vuelve a esta primera etapa como un momento crítico para lograr una buena alimentación y por ende un aporte de nutrientes necesarios para formar adecuadamente su cerebro, a la vez se convierte en una única oportunidad para lograr garantizar el desarrollo de todo su potencial en la edad adulta. La alimentación de ese niño durante sus primeros 5 años determinará no sólo su futuro personal, sino el de su propia familia y el de su país (13).

Los resultados de la presente investigación son una información valiosa sobre el consumo de los multimicronutrientes y la forma como podrían mejorarse, ya que a la fecha no han disminuido los índices de anemia en los niños; Basados en la información proporcionada, producto de la presente investigación; se tendrán datos exactos sobre cuáles son las estrategias que deben abordar las instituciones encargadas en aspectos de salud, como son el MINSA a través del Centro de Salud del lugar e incluso la Municipalidad del Distrito; a través de sus diversos programas.

El objetivo del presente trabajo es el mejorar los conocimientos, actitudes y prácticas del consumo de multimicronutrientes en niños menores de 3 años, de la Micro Red de Salud Chilca, mediante una intervención educativa.

\section{MATERIAL Y MÉTODOS}

En el presente trabajo se utilizó el método científico (14), siendo una investigación de tipo aplicada (15), nivel aplicativo (15) y con un diseño epidemiológico (16), experimental, dentro del grupo de los ensayos comunitarios.

La unidad de análisis fue el niño menor de 3 años, que asiste a los Centros de Salud de la Micro Red Chilca. La unidad de información fue la madre, padre o cuidador (a) del niño. La población está constituida por 1200 niños y niñas menores de 3 años que asisten al Centro de Salud de la Micro Red Chilca, con una muestra de 65 niños y niñas, cuyos padres aceptaron voluntariamente participar en el estudio y firmaron el consentimiento informado, por lo que se aplicó un muestreo no probabilístico, intencionado; considerando los criterios de inclusión y exclusión. La técnica de recolección de datos para la variable "conocimientos, actitudes y prácticas" es la entrevista y el instrumento aplicado un cuestionario estructurado y que pasó por pruebas de confiabilidad y validez a través de expertos en el tema. Para la variable intervención educativa, se capacitó al personal de campo en estrategias y metodologías educativas, basando sus contenidos en el diagnóstico inicial, con mensajes de comunicación $\mathrm{y}$ visitas domiciliarias.

Luego de haber recolectado los datos, por medio de la aplicación de los instrumentos seleccionados, se diseñó una base de datos inicial en el programa Excel versión 2013. Para realizar la prueba de hipótesis se utilizó el programa estadístico IBM-SPSS, versión 24; a través de la prueba estadística t de Student.

\section{RESULTADOS}

Se presentan los resultados considerando los hallazgos encontrados en la fase diagnóstica (pre-test) y los que se encontraron después de la intervención (post-test), con la intención de evidenciar el impacto que ha producido el trabajo realizado por los investigadores del estudio. En la tabla 1, se presentan los resultados sobre los conocimientos del consumo de multimicronutrientes; ha sido necesario averiguar el tipo de suplemento que están recibiendo los niños, se observa que el $72,3 \%$ de ellos está consumiendo el micronutriente en polvo y el $27,7 \%$ recibe el jarabe. Esta información fue necesaria para la orientación que recibieron los padres/- madres/cuidadores de los niños, ya que cada producto tiene indicaciones y especificaciones diferentes, según las normas técnicas.

En la tabla 1 se observa que han mejorado sus conocimientos con respecto, al por qué su niño (a) consume los suplementos; ya que antes de la 
Tabla 1. Conocimientos sobre el consumo de multimicronutrientes en niños menores de 3 años, de la Micro Red de Salud Chilca -2017 .

\begin{tabular}{|c|c|c|c|c|}
\hline & f & $\%$ & f & $\%$ \\
\hline \multicolumn{5}{|c|}{ ¿Qué tipo de suplemento está recibiendo su niño? } \\
\hline Micronutriente en polvo & 47 & 72,3 & 47 & 72,3 \\
\hline Jarabe (sulfato ferroso) & 18 & 27,7 & 18 & 27,7 \\
\hline No recibe ningún suplemento & 0 & 0,0 & 0 & 0,0 \\
\hline \multicolumn{5}{|c|}{ ¿Para qué cree que su niño(a) consume los suplementos? } \\
\hline Para curarse de la anemia & 24 & 36,9 & 21 & 32,3 \\
\hline Para prevenir la anemia & 32 & 49,2 & 42 & 64,6 \\
\hline Para no enfermarse frecuentemente & 5 & 7,7 & 2 & 3,1 \\
\hline Para crecer & 2 & 3,1 & 0 & 0,0 \\
\hline No sabe / no contesta & 2 & 3,1 & 0 & 0,0 \\
\hline \multicolumn{5}{|c|}{ ¿Con qué frecuencia se debe consumir el suplemento? } \\
\hline 1 vez al dia & 45 & 69,2 & 62 & 95,4 \\
\hline 2 veces al dia & 15 & 23,1 & 3 & 4,6 \\
\hline 3 veces al dia & 1 & 1,5 & 0 & 0,0 \\
\hline 4 veces al dia & 2 & 3,1 & 0 & 0,0 \\
\hline 5 veces al dia & 2 & 3,1 & 0 & 0,0 \\
\hline \multicolumn{5}{|c|}{ ¿En qué momento se debe dar el suplemento? } \\
\hline Antes de las comidas & 8 & 12,3 & 10 & 15,4 \\
\hline Junto con las comidas & 47 & 72,3 & 53 & 81,5 \\
\hline Inmediatamente después de las comidas & 5 & 7,7 & 2 & 3,1 \\
\hline Una o dos horas después de las comidas & 4 & 6,2 & 0 & 0,0 \\
\hline No sabe / no contesta & 1 & 1,5 & 0 & 0,0 \\
\hline \multicolumn{5}{|c|}{ Si su niño dejó de consumir el suplemento durante mucho tiempo ¿puede volver a recibir el tratamiento? } \\
\hline $\mathrm{Si}$ & 33 & 50,8 & 46 & 70,8 \\
\hline No & 21 & 32,3 & 17 & 26,2 \\
\hline No sabe / No contesta & 11 & 16,9 & 2 & 3,1 \\
\hline
\end{tabular}

intervención se indicaba que el $49,2 \%$ era para prevenir la anemia y post intervención se incrementó a un $64,6 \%$; siendo esto un aspecto de vital importancia para esperar que las madres de familia tomen interés en realizar acciones basadas en la comprensión del problema.

Asimismo, mejoraron sus conocimientos con respecto a la frecuencia del consumo del suplemento en los niños(as) menores de 3 años; previo a la intervención solo el $69,2 \%$ manifestaba que era a diario y luego de la intervención el 95,4\% respondía acertadamente la pregunta.

También mejoraron sus conocimientos con respecto al momento en que deben brindar el suplemento, que según la norma es con las comidas, mezclando bien las 2 cucharas de comida separada con los micronutrientes; los datos obtenidos antes de la intervención indicaban que sólo el 72,3\% sabía con precisión en qué momento dar el suplemento, este porcentaje subió a 81,5\% luego de la intervención.

También se observaron cambios en relación a sus conocimientos sobre el reinicio del suplemento; antes de la intervención solo el 50,8\% indica que puede volver a recibir el tratamiento, tiempo después de haberlo dejado y luego de la intervención la cifra se incrementó hasta el 70,8\%.

Este cambio es fundamental ya que en muchos casos existe la creencia equivocada que, si se dejó algunos días de consumir el micronutriente, ya no se puede continuar después. Sus conocimientos con respecto al momento en que deben brindar el suplemento; también evidenció mejoras, antes de la intervención el 
$60,0 \%$ indica que el suplemento se debe dar mezclado con segundos, purés o papillas y post intervención el porcentaje se incrementó al $81,5 \%$.

Los padres han mejorado sus conocimientos con respecto a los problemas que presentan los niños cuando tienen anemia; antes de la intervención el $50,8 \%$ indica que en el futuro tendrá bajo rendimiento en la escuela y post intervención el porcentaje se incrementó al 81,5\%; también se puede observar que antes de la intervención el 27,7\% indica que aumenta el sueño y post intervención este porcentaje se disminuyó a $10,8 \%$; este aspecto es importante ya que se espera que la familia se involucre en el tema, entendiendo los grandes problemas que tiene la anemia.

Con relación a las actitudes sobre el consumo del multimicronutriente (tabla 2), en un inicio solo el $61,5 \%$ de padres/madres/cuidadores señalan que consideran que es bueno para el niño consumir el micronutriente; pero luego de la intervención esta cifra aumentó a 92,3\%. Un tema trascendental en las actitudes es que los miembros de la familia consideren que es difícil el consumo del micronutriente; por esta razón se trabajó el tema y se observa que mejoraron sus actitudes con respecto a la dificultad para brindar el suplemento, en la evaluación diagnóstica el 7,7\% indicaba que es difícil brindar el suplemento y después de la intervención el porcentaje disminuyó al 4,6\%; este cambio positivo también se demostró en el primer ítem de que consideran que no es difícil brindarle el suplemento.

Los padres también han mejorado sus actitudes con respecto a que si siente que está haciendo bien en darle el suplemento todos los días a su niño(a); ya que antes de la intervención se reporta que el 58,5\% manifiesta que sí y post intervención el porcentaje se

Tabla 2. Actitudes sobre el consumo de multimicronutrientes en niños menores de 3 años, de la Micro Red de Salud Chilca - 2017

\begin{tabular}{|c|c|c|c|c|}
\hline & \multicolumn{2}{|c|}{ Pre-test } & \multicolumn{2}{|c|}{ Post Test } \\
\hline & f & $\%$ & f & $\%$ \\
\hline \multicolumn{5}{|c|}{ ¿Qué tan bueno es para su niño (a) consumir el suplemento } \\
\hline No es bueno & 3 & 4,6 & 1 & 1,5 \\
\hline No estoy seguro que sea bueno & 19 & 29,2 & 2 & 3,1 \\
\hline Es bueno & 40 & 61,5 & 60 & 92,3 \\
\hline En algunos niños puede ser bueno & 3 & 4,6 & 2 & 3,1 \\
\hline \multicolumn{5}{|c|}{ ¿Qué tan dificil es darle el suplemento todos los dias a su niño? } \\
\hline No es dificil & 35 & 53,8 & 38 & 58,5 \\
\hline Regular & 23 & 35,4 & 23 & 35,4 \\
\hline No lo sé & 2 & 3,1 & 1 & 1,5 \\
\hline Es dificil & 5 & 7,7 & 3 & 4,6 \\
\hline \multicolumn{5}{|c|}{ ¿Siente que hace bien en darle el suplemento todos los dias a su niño? } \\
\hline Si / Segura & 38 & 58,5 & 58 & 89,2 \\
\hline Regular / No lo sé & 25 & 38,5 & 6 & 9,2 \\
\hline No / Insegura & 2 & 3,1 & 1 & 1,5 \\
\hline \multicolumn{5}{|c|}{ ¿Cree que la dosis que recibe su niño(a) es: ? } \\
\hline Suficiente & 43 & 66,2 & 59 & 90,8 \\
\hline Insuficiente & 12 & 18,5 & 4 & 6,2 \\
\hline Mucho & 4 & 6,2 & 2 & 3,1 \\
\hline No sabe / No contesta & 6 & 9,2 & 0 & 0,0 \\
\hline \multicolumn{5}{|c|}{ ¿Qué tan grave cree que es no darleel suplemento a su niño(a)? } \\
\hline No es grave & 15 & 23,1 & 18 & 27,7 \\
\hline No lo sè & 28 & 43,1 & 12 & 18,5 \\
\hline Es grave & 17 & 26,2 & 35 & 53,8 \\
\hline No sabe / No contesta & 5 & 7,7 & 0 & 0,0 \\
\hline
\end{tabular}


Tabla 3. Prácticas sobre el consumo de multimicronutrientes en niños menores de 3 años, de la Micro Red de Salud Chilca - 2017.

\begin{tabular}{lcccc}
\hline & \multicolumn{2}{c}{ Pre-test } & \multicolumn{2}{c|}{ Post Test } \\
& f & \% & f & \% \\
\hline ¿Su niño consumió el suplemento el dia de ayer? & & & & \\
Si & 28 & 43,1 & 48 & 73,8 \\
No & 37 & 56,9 & 17 & 26,2 \\
En el último mes: ¿Dejó de darle el suplemento algun dia? & & & \\
Si & 32 & 49,2 & 32 & 49,2 \\
No & 30 & 46,2 & 33 & 50,8 \\
No sabe / No contesta & 3 & 4,6 & 0 & 0,0 \\
¿Siente que hace bien en darle el suplemento todos los dias a su niño (a)? & & & & \\
Con segundos, purés o papillas & 46 & 70,8 & 55 & 84,6 \\
Con sopas & 8 & 12,3 & 1 & 1,5 \\
Con jugos & 6 & 9,2 & 8 & 12,3 \\
Con agua & 4 & 6,2 & 1 & 1,5 \\
No sabe/ No contesta & 1 & 1,5 & 0 & 0,0 \\
Se presentó molestias el niño(a); suprimió el tratamiento? Suficiente & & & & \\
Si se suprimió el tratatmiento & 23 & 35,4 & 7 & 10,8 \\
No se suprimió el tratamiento & 32 & 49,2 & 28 & 43,1 \\
No ha presentado molestias & 10 & 15,4 & 30 & 46,2 \\
\hline
\end{tabular}

Tabla 4. Estadísticas de muestras emparejadas

\begin{tabular}{llcccc}
\hline & Media & $\mathrm{n}$ & $\begin{array}{c}\text { Desviación } \\
\text { estandar }\end{array}$ & $\begin{array}{c}\text { Media de error } \\
\text { estandar }\end{array}$ \\
\hline Par 1 & Nota del pre-test & 7,94 & 65 & 3,015 & 0,374 \\
& Nota del post-test & 11,14 & 65 & 1,802 & 0,223 \\
\hline
\end{tabular}

Tabla 5. Prueba de muestras emparejadas

\begin{tabular}{|c|c|c|c|c|c|c|c|c|c|}
\hline \multicolumn{10}{|c|}{ Diferencias emparejadas } \\
\hline & & \multirow[t]{2}{*}{ Media } & \multirow[t]{2}{*}{$\begin{array}{l}\text { Desviación } \\
\text { estandar }\end{array}$} & \multirow[t]{2}{*}{$\begin{array}{l}\text { Media } \\
\text { de error } \\
\text { estandar }\end{array}$} & \multicolumn{2}{|c|}{$\begin{array}{c}95 \% \text { de intervalo } \\
\text { de confianza de la } \\
\text { diferencia }\end{array}$} & \multirow[t]{2}{*}{$\mathrm{t}$} & \multirow[t]{2}{*}{$\mathrm{gl}$} & \multirow[t]{2}{*}{$\begin{array}{c}\text { Sig. } \\
\text { (bilateral) }\end{array}$} \\
\hline & & & & & Inferior & Superior & & & \\
\hline \multirow{3}{*}{ Par 1} & Nota del pre-test & & & & & & & & \\
\hline & & $-3,200$ & 3,496 & 0,434 & $-4,066$ & $-2,334$ & $-7,379$ & 64 & 0,000 \\
\hline & Nota del post-test & & & & & & & & \\
\hline
\end{tabular}

incrementó al 89,2\%; también se pudo observar que antes de la intervención el $38,5 \%$ indica que es regular y post intervención este porcentaje se disminuyó a $9,2 \%$; asimismo se puede observar que antes de la intervención el 3,1\% de los entrevistados desconocía o no respondían esta pregunta sin embargo post la intervención solo el 1,5\% no responde o está inseguro.
Todo esto ayuda a mejorar las actitudes sobre el consumo del micronutriente.

Los padres han mejorado sus actitudes con respecto a la dosis de suplemento que recibe el niño; ya que antes de la intervención se reporta que el $66,2 \%$ manifiesta que es suficiente y post intervención el 
porcentaje se incrementó al 90,8\%; también se puede observar que antes de la intervención el 18,5\% indica que es insuficiente y post intervención este porcentaje se disminuyó a $6,2 \%$. También se observan mejoras en sus actitudes con respecto a qué tan grave es no darle el suplemento a su niño(a); se puede observar que antes de la intervención el 43,1\% indica que no sabe y post intervención este porcentaje se disminuyó a $18,5 \%$; asimismo se puede percibir que antes de la intervención el 26,2\% de los entrevistados indicaban que no darle el suplemento a su niño era grave y post intervención se incrementó a 53,8\%. habiéndose logrado uno de los objetivos de la intervención.

Con relación a las prácticas sobre el consumo del multimicronutrientes (Tabla 3), se observa que los padres/madres/cuidadores han mejorado sus prácticas con respecto a que si el niño(a) consumió el suplemento el día anterior a la visita; ya que antes de la intervención se reporta que solo el 43,1\% consumía el suplemento y post intervención el porcentaje se incrementó a $73,8 \%$; esta pregunta está relacionada al interés que mostraron los miembros de la familia para garantizar que el niño consuma el suplemento a partir de la consejería brindada. La continuidad del consumo, también brindó resultados positivos (46,2\% antes de la intervención y 50,8\% post intervención).

Es necesario precisar que en las capacitaciones se hizo énfasis en indicar que no se debe compartir el sobre de Micronutrientes con la comida de otro miembro del hogar ni tampoco con otro niño. también han mejorado sus prácticas con respecto al consumo; antes de la intervención se reporta que el $70,8 \%$ de los niños(as) menores de 3 años lo consumen con segundos, purés o papillas y post intervención el porcentaje se incrementó a $84,6 \%$; también se tiene como hallazgo antes de la intervención que el $12.3 \%$ lo consumen con sopas y post la intervención solo el 1,5\%; asimismo se observa que antes de la intervención el 6.2\% lo consumía solo con agua y post la intervención este porcentaje también disminuyó al 1,5\%, entendiendo que las prácticas negativas han disminuido, incrementándose las positivas.

Los padres también mejoraron sus prácticas con respecto a que sí suprimieron el tratamiento cuando sus niños (as) presentaban molestias; antes de la intervención se suprimía en un $35,4 \%$ y post la intervención solo el 10,8\%. Se hizo especial énfasis en mensajes como el que el niño puede presentar algunos malestares que van a pasar y que no hay por qué alarmarse, además lo capacitadores enseñaron las formas cómo se deben de manejar las molestias que se presentan tras el consumo del suplemento.
La prueba de hipótesis se realizó asignando una puntuación a los cuestionarios aplicados, antes (pre-test) y después (post-test) de la intervención educativa, con el fin de comparar los resultados y aplicar una prueba estadística que nos confirme la aceptación de la hipótesis planteada. Para la elección de la prueba de hipótesis se tuvo que seleccionar el estadístico de prueba, para lo cual se utilizaron los criterios relacionados al tipo de investigación, nivel, diseño, objetivos estadísticos, escalas de medición de las variables y el comportamiento de los datos; razón por la cual se eligió la prueba estadística paramétrica t-Student para muestras relacionadas. Previamente a la aplicación de la prueba estadística se ejecutó la prueba de normalidad de los datos obtenidos del test aplicado a las madres a través de Kolmogorov- Smirnov.

Para tomar la decisión estadística de rechazar la hipótesis nula y aceptar la de investigación, se consideró el nivel de significancia del 95\% y de 64 grados; la lectura del p-valor se utilizó para determinar la aceptación de la hipótesis de investigación si resultó menor a 0,05 , la hipótesis de investigación afirma que los conocimientos, actitudes y prácticas del consumo de multimicronutrientes en niños menores de 3 años, cambian positivamente, después de la intervención educativa.

A partir de los resultados que se presentan en las tablas 4 y 5 , se puede concluir que se rechaza la hipótesis nula y se acepta la hipótesis de investigación o alterna, por lo tanto: Los conocimientos, actitudes y prácticas del consumo de multimicronutrientes en niños menores de 3 años, cambian positivamente, después de una intervención educativa.

\section{DISCUSIÓN}

En la actualidad los programas que están siendo utilizados por diferentes gobiernos en el mundo como estrategia prioritaria para disminuir los índices de déficit de micronutrientes en niños es el de la suplementación; ya que permiten alcanzar de manera focalizada a los grupos de alto riesgo y proporcionar el aporte de elementos como el hierro, zinc, y vitaminas para corregir la deficiencia de manera rápida y efectiva; pero en el Perú no ha sido posible lograr estos resultados, a partir de dificultades propias de la idiosincrasia peruana, además de barreras técnicas y otros problemas como las dificultades de nuestra geografía como la distribución, falta de una red de información sobre el uso y beneficios de estos micronutrientes, un consumo bajo de parte de los niños y la aparición de los efectos secundarios; propios de 
suplementar a niños desnutridos en su mayoría. Pero lo que sí queda claro es que se reconoce de forma irrefutable una creciente necesidad de contar con evidencia científica para tomar decisiones y orientar la forma de cómo debe ser el diseño e implementación de los programas de suplementación nutricional, específicamente el de micronutrientes.

Los resultados del presente trabajo muestran que la capacitación e información brindada a las madres con niños que reciben los micronutrientes tuvo un efecto positivo en los conocimientos, actitudes y prácticas. Basta observar los cambios obtenidos entre la fase de diagnóstico inicial y la fase final de evaluación de los cambios en intervención educativa, para ver que se logró mejorar los conocimientos, actitudes y prácticas sobre el consumo de multimicronutrientes, en los niños menores de 3 años de la Micro Red de Salud Chilca; un aspecto importante a considerar es el marco conceptual que se utilizó para esta intervención y la estrategia de visitas domiciliarias como el eje de todo el proceso, por lo que se permitió evaluar in situ los problemas o barreras que se presentaban; aunque es necesario hacer un análisis de cada uno de los componentes sobre los que se investigó.

Con relación a los resultados se ha podido encontrar que inicialmente un $14 \%$ de las madres no tenían conocimiento del propósito del consumo del micronutriente, en algunos casos creían que era para crecer y en otros casos para no enfermarse o no sabían para qué; en el estudio de Solano (17), se indica que el $11 \%$ de las madres no saben para que se administran los micronutrientes; frente a estas cifras se ha podido evidenciar una mejora en el tema, ya que luego de la intervención solo el 3,1\% desconocía el objetivo de darle al niño el suplemento; este aspecto es trascendental ya que todo actividad o práctica se vuelve sostenible a partir de comprender primero e interiorizar después los motivos reales por la que se debe consumir un determinado suplemento y esto a la vez se vuelve un elemento valioso para las intervenciones, ya que nos permite identificar el nivel de conocimiento que posee la población, a fin de implementar actividades educativas orientadas a promover el aprendizaje a través de la educación (18), esto coincide con autores como Rocha (19), que afirman que el conocimiento ha sido visto como uno de los más importantes recursos de una organización por ser capaz de tornar las acciones, en los planes organizacionales, lo que incluye en la actualidad a los sistemas de salud y los hace más inteligentes, eficientes y eficaces.
La frecuencia con la que se debe consumir el micronutriente es una información básica para su efectividad, al término de la intervención, el 95,4\% precisaba con certeza la frecuencia adecuada para el consumo, esto es importante ya que en otros estudios realizados (20), se ha encontrado datos similares a los que se halló antes de la intervención (66,7\%); esto corrobora que la consejería se convierte en un medio importante para mejorar los conocimientos y las prácticas, pero que debe hacerse en función a un diagnóstico adecuado que permita comprender la problemática y actuar de forma certera y asertiva.

En este tema específico es necesario que se cumpla con la dosis del suplemento, ya que es la única forma de garantizar su eficacia para disminuir los niveles de anemia en los niños, si se cumple con la dosificación, ya que la OMS (21) reporta que se ha comprobado que la administración de suplementos de hierro aumenta eficazmente las concentraciones de hemoglobina en niños, especialmente en los que sufren de anemia. Otro aspecto importante, es el momento de administrar el suplemento al niño, este se mejoró luego de la intervención, según la Norma Técnica vigente se recomienda que para la administración del micronutriente no necesitan cocinarse, solo deben ser añadidos en una ración de comida espesa de los bebés, ya sea en papillas, purés o segundos, se debe mezclar el contenido del sobre de Micronutrientes con 2 cucharadas de la comida, cuando se encuentre a temperatura que el niño ya pueda comerla (21); por lo tanto luego, de la intervención el $81,5 \%$ de las madres respondieron acertadamente con esta respuesta; lo que garantiza que las prácticas podrían mejorar también. Además de otros conocimientos que la madre demostró mejorar como el hecho de que si su niño dejó de consumir el suplemento durante mucho tiempo podía volver a recibir el tratamiento con las especificaciones seguidas en la referida norma técnica. Fue necesario investigar sobre los conocimientos que tienen las madres o cuidadoras de los niños respecto a los problemas que presenta un niño cuando tiene anemia, por ser esto un factor determinante para el interés y las actitudes que tenga respecto a la adherencia al micronutriente; en este aspecto la intervención educativa logró un cambio significativo en más de $31 \%$, que contestaron que el principal problema es el bajo rendimiento del niño en la escuela; Pollitt (3), indican que el desarrollo cognitivo es el principal problema de los niños anémicos, y Zavaleta además concluye en su estudio indicando que estos niños estarían en riesgo de no alcanzar su potencial de desarrollo infantil a causa de esta enfermedad y que se hace necesario, fortalecer las acciones para alcanzar una mayor adherencia y 
efectividad de la suplementación con hierro a la madre y al niño, promover la alimentación adecuada tanto en el niño como en la gestante y mejorar la atención sanitaria para reducir la carga de enfermedad infantil y así contribuir a prevenir la anemia (22). Estudios como el de Guzmán (23) nos reportan que la anemia en la infancia además afecta al crecimiento y al desarrollo de los niños, disminuye la resistencia a las infecciones y altera el desarrollo cognitivo y psicomotor. Por lo tanto, es importante que las madres manejen de forma correcta esta información ya que incide en el interés que pueda poner en el tema.

Con relación a las actitudes que tienen las madres sobre los micronutrientes, El interés de los científicos por el estudio de las actitudes se ha enfocado a su relación con los comportamientos (24); surgiendo una interrogante difícil de responder como ¿en qué medida evaluar las actitudes ayudan a predecir comportamientos?; tal sería la cuestión sobre la que han girado el presente trabajo para investigar aspectos como ¿qué tan bueno es consumir el micronutriente?, ¿qué tan difícil es darle al niño? o preguntarle a la madre si siente que está haciendo bien el darle el suplemento al niño; desde esta perspectiva, la actitud aparece tan estrechamente ligada al comportamiento como esa "respuesta implícita" que tan sólo aguarda la presencia de la situación estimuladora para pasar a ser respuesta manifiesta, que para nuestro caso sería la práctica. No se evidenciaron cambios muy significativos (después de la intervención) en la relacionado a lo que las madres consideran que no es tan difícil darle el micronutriente al niño $(58,5 \%)$, este hecho puede estar relacionado a que estudios sobre el tema como el de Vargas (25), consideran que las actitudes tienden a permanecer bastante estables con el tiempo y que son las predisposiciones a responder de una determinada manera con reacciones favorables o desfavorables hacia algo.

Las integran las opiniones o creencias, los sentimientos y las conductas, factores que a su vez se interrelacionan entre sí; por lo que resulta difícil cambiarlos en un periodo corto de tiempo. Sin embargo, se notó un incremento significativo en la actitud de las madres respecto a que sentían que estaban haciendo bien en darle el suplemento a su hijo, es así que al final de la intervención el 89,2\% consideró positivo el consumo del micronutriente; este cambio corrobora lo investigado por Junco (26) quien indica que este componente actitudinal debe hacer énfasis en las relaciones que existen entre la comunicación educativa, el mejoramiento de prácticas, comportamientos y las mismas actitudes, y los resultados de impacto y proceso. Se fundamenta en el concepto de que el incremento de conocimiento e información contribuye al mejoramiento de prácticas de cuidado de la madre y el niño, estimula el compromiso para dar lo mejor de su capacidad, puede atraer la atención de los otros miembros del hogar, y moviliza recursos y apoyo dentro y fuera del entorno del niño. Las actitudes orientan los actos, si las influencias externas sobre lo que se dice o hace tienen una mínima incidencia. También los orientan si la actitud tiene una relación específica con la conducta, a pesar de lo cual la evidencia confirma que, a veces, el proceso acostumbra a ser inverso y los actos no se corresponden.

Uno de los aspectos que se han mejorado a través de la intervención está relacionado al consumo frecuente del micronutriente, inicialmente se encontró que solo el $43,1 \%$ consumió el suplemento el día anterior a la visita, pero al final de la intervención, el $73,8 \%$ lo había hecho; sin embargo, hay un gran porcentaje que no está consumiendo el producto de forma diaria como lo estipula la norma técnica, cifras similares se reportan en los estudios realizados por investigadores como Huamán (27), quien encontró que el 69,6\% de los que recibieron la intervención no la consumieron en forma adecuada.

Para evaluar esta situación por la que están atravesando las familias en el Perú y en particular en las que asisten a la Micro Red De Salud Chilca, en Huancayo; hay que considerar varios aspectos que pueden estar incidiendo, en primer lugar, tener en cuenta que a pesar de que los suplementos tipo micronutrientes tienen una mejor adherencia que otros tratamientos con sulfato ferroso, puede ocurrir una gran variabilidad en programas de uso masivo (como el que se maneja en Perú), donde es relevante y necesario el monitorear la aceptabilidad y uso por parte de los niños, conocer los motivos por los que no lo consumió, para que tengamos en cuenta las barreras que están influyendo y de esta forma para mitigarlo y supervisarlo en forma continua, como se ha demostrado en estudios realizados en otros países (28).

Al respecto, se evaluaron diferentes estrategias para lograr una mayor adherencia, como incrementar el tiempo de exposición, pero con dosis flexibles para asegurar cubrir la deficiencia de hierro (29), o tener solo una toma semanal, pero a altas dosis, situaciones que podrían ser evaluadas en nuestro contexto, no solo 
en Huancayo, sino en otras regiones de nuestro país. Otro asunto que es un factor determinante para el consumo del micronutriente lo constituye las diversas molestias que reportan los niños y que ocasionan el abandono del tratamiento, en el estudio de Chilca se ha tenido en consideración este aspecto, por lo que antes de la intervención el 35,4\% de las madres suspendió el tratamiento cuando el niño presentó molestias, pero al final de la intervención ese porcentaje disminuyó a $10,8 \%$, este avance es significativo, ya que si contrastamos con otros estudios como el de Hinostroza (30), encontró una baja adherencia al consumo entre otros factores porque las madres manifestaban que el estreñimiento fue el efecto colateral que más presentó su niño, conjuntamente con las heces oscuras; aunque explicaron que este rechazo no fue de inmediato, sino días o semanas después del primer sobre administrado, indicando que otro problema fue el sabor que tenía; pero el estudio de Gutiérrez (31), evaluó una particularidad importante en este tema; concluyó que los niños y niñas que no presentaron malestares desde el inicio se mantuvieron hasta el final del seguimiento, sin embargo, aumentaron los niños que presentan malestares de vez en cuando en vista que la frecuencia de suplementación que se les brindaba fueron de manera interdiaria o una vez por semana. Similar fue en niños que si presentaban los malestares.

Si bien es cierto en el trabajo se puede evidenciar que, si es posible mejorar los conocimientos, actitudes y prácticas respeto al consumo del micronutriente en los niños, una de las limitaciones que se han presentado en la Micro Red de Salud Chilca, es que no se ha evaluado el nivel de adherencia; ya que en publicaciones de la Organización Mundial de la Salud (32) se considera que no existe un "patrón de oro" para medir el comportamiento de adherencia, consideramos que para la determinación de la adherencia en micronutrientes, el empleo del recuento de los sobres consumidos puede ser una estrategia (según la norma técnica vigente en el Perú), recomendado tanto en la visita domiciliaria como en el consultorio.

Sin embargo, los errores de recuento son comunes y dan lugar de manera característica a la sobreestimación del comportamiento, lo que puede ser complementado con otros instrumentos como pruebas estandarizadas. Lo concreto para la determinación ideal de la adherencia terapéutica es que el niño consuma el 100\% del suplemento, pero resulta poco práctico, se han ensayado para el caso de los micronutrientes puntos de corte desde el $75 \%$ del consumo.

\section{CONCLUSIONES}

Los conocimientos, actitudes y prácticas sobre el consumo de multimicronutrientes, cambiaron positivamente después de la intervención educativa ( $\mathrm{p}$-valor $=0,000$ ), en los niños menores de 3 años de la Micro Red de Salud Chilca de la provincia de Huancayo. Los cambios más significativos con relación a los conocimientos sobre el consumo de multimicronutrientes en los niños menores de 3 años, se dio en lo relacionado a la información que manejaban las madres sobre los principales problemas que presenta un niño cuando presenta anemia, reconociendo entre otras limitantes el bajo rendimiento escolar que tendrá el niño en el sistema escolar.

Con relación a las actitudes sobre el consumo de multimicronutrientes en los niños menores de 3 años, se evidenció mejoras en el hecho que al final de la intervención se logró tener una actitud favorable de las madres, respecto a lo bueno que resulta para el niño consumir el suplemento. Las mejoras en las prácticas sobre el consumo de multimicronutrientes en los niños menores de 3 años, se evidenció en lo relacionado al consumo del suplemento el día anterior a la visita domiciliaria, logrando un incremento de $30,7 \%$ con relación al resultado inicial.

Se recomienda que el gobierno establezca estrategias de intervención para mejorar el consumo de los micronutrientes, a partir de diagnósticos iniciales, que nos puedan proporcionar información sobre la situación actual del problema y las limitantes que impiden que el niño consuma adecuadamente el producto; donde se consideren las actitudes, como un elemento fundamental para comprender y mejorar las prácticas del consumo.

Es necesario combinar más de una estrategia para reforzar los mensajes brindados en la consejería e incrementar el impacto a través de las visitas domiciliarias, las mismas que deben de constituirse en el eje de toda la intervención.

Conflicto de Intereses: Los autores de este artículo declaran que no tienen ningún conflicto de intereses.

\section{Correspondencia:}

Dra. Q.F. Diana Andamayo Flores.

Correo electronico: dandamayo@uroosevelt.edu.pe 


\section{REFERENCIAS BIBLIOGRÁFICAS}

1. Organización Mundial de la Salud. Prevalencia mundial de la anemia y número de personas afectadas. Ginebra: Organización Mundial de la Salud ; 2017. (Citado en junio del 2018) Disponible en: http:// www.who.int/vmnis/database/anaemia/anaemia data status $\mathrm{t} 2 / \mathrm{es} /$

2. Instituto Nacional de Estadística e informática. Encuesta demográfica de Salud Familiar 2016. Lima: Instituto Nacional de Estadística e informática; 2017.

3. Pollitt E. Desnutrición, pobreza e inteligencia. 2da ed. Perú: editorial Universitaria; 2007.

4. Logan C, Sguassero Y, Cuesta C. Anemia y adherencia a la suplementación oral con hierro en una muestra de niños usuarios de la red de salud pública de Rosario, Santa Fe. Arch argent pediatr. 2013; 111(4): 288-294.

5. Aguirre J, Chávez A, Medina B, García E, Carrasco M, Guarneros N. Impacto del suministro de harina de maíz fortificada en la anemia de preescolares de zonas indígenas de México. Gac Sanit.2013; 27(6): 541-544. (Citado 1 de noviembre 2017) Disponible en: http://scielo.isciii.es/scielo.php?script=sci_arttext \&pid=S021391112013000600013\&lng=es.http:// dx.doi.org/10.1016/j.gaceta.2013.04.013.

6. Vizuet N, Shamah T, Gaona E, Cuevas L, Méndez I. Adherencia al consumo de los suplementos alimenticios del programa PROSPERA en la reducción de la prevalencia de anemia en niños menores de tres años en el estado de San Luis Potosí, México. Nutr Hosp. 2016 ; 33 (4): 782-789. (Citado 1 de noviembre 2017) Disponible en: http://scielo. isciii.es/scielo. php?script $=$ sci_arttext\&pid $=\mathrm{S} 0212$ $16112016000400004 \& \operatorname{lng}=$ es.http://dx.doi. org/10.20960/nh.370.

7. Vargas A, Bado R, Alcázar L, Aquino O, Rodríguez A, Novalbos JP. Efecto de un suplemento nutricional a base de lípidos en los niveles de hemoglobina e indicadores antropométricos en niños de cinco distritos de Huánuco, Perú. Rev Perú Med Exp Salud Pública. 2015; 32(2): 237-44.

8. Munayco CV, Ulloa ME, Medina J, et al. Evaluación del impacto de los multimicronutrientes en polvo sobre la anemia infantil en tres regiones andinas del Perú. Rev Perú Med Exp Salud Pública. 2013; 30(2): 229-34.

9. Chamorro J, Torres K. Efecto de la suplementación con multimicronutriente y estado nutricional en niños menores de tres años en comunidades de Huando y Anchonga - Huancavelica. Tesis de Grado. Lima, Perú: Universidad Nacional Mayor de San Marcos; 2010. 44 p.

10. Durand D. Relación entre el nivel de conocimientos que tienen las madres sobre alimentación infantil y el estado nutricional de niños preescolares en la
Institución Educativa Inicial No 111 - Callao en el año 2008. Tesis de Grado. Lima, Perú: Universidad Nacional Mayor de San Marcos; 2010.

11. Ministerio de Salud. Resolución Ministerial No 250-2017-MINSA. Lima: Ministerio de Salud; 2017.

12. Munares O, Gómez G. Adherencia a multimicronutrientes y factores asociados en niños de 6 a 35 meses de sitios centinela, Ministerio de Salud, Perú. Rev bras epidemiol. 2016; 19(3):539-553. doi:: http://dx.doi.org/10.1590/1980-5497201600030006.

13. Sierra L. Promoción del crecimiento y desarrollo en la primera infancia. Lima: Fondo de las Naciones Unidas Para La Infancia; 2013.

14. Ñaupas H, Mejía E, Novoa E, Villagómez A. Metodología de la investigación. Bogota, Colombia: Ediciones de la U; 2014.

15. Supo J. Seminarios de investigación científica. Lima: Editorial Bioestadístico EIRL; 2014.

16. Villa A, Moreno L, García G. Epidemiología y estadística en salud pública. Madrid, España: McGraw Hill / Interamericana; 2011.

17. Solano L. Conocimientos y prácticas que tienen las madres sobre los multimicronutrientes que reciben sus niños en un centro de salud, 2016. Lima, Perú: Universidad Nacional Mayor de San Marcos; 2016.

18. Huayaney D. Conocimiento de las madres sobre la prevención de la anemia ferropénica en la estrategia de CRED en el Centro de Salud de Chasquitambo. Tesis de posgrado. Lima: Universidad Nacional Mayor De San Marcos; 2013.

19. Rocha ESB, Nagliate P, Furlan CEB, Rocha K, Trevizan MA, Mendes IAC. Gestión del conocimiento en salud: revisión sistemática de la literatura. Rev. Latino-Am. Enfermagem. $2012 ; 20$ (2):0-0. (Citado 1 de noviembre 2017) Disponible en: http://www. scielo.br/pdf/rlae/v20n2/es_24.pdf

20. Carmen A, Carrasco A, Coronel F. Factores que condicionan el consumo de multimicronutrientes según el cuidador primario, 2017. Tesis de Grado. Lima, Perú: Universidad Peruana Cayetano Heredia; 2018.

21. Ministerio de Salud del Perú. Norma técnica manejo terapéutico y preventivo de la anemia en niños, adolescentes, mujeres gestantes y puérperas. Resolución Ministerial N 958-2012/MINSA. Perú: Ministerio de Salud del Perú; 2017.

22. Zavaleta N, Astete-Robilliard L. Efecto de la anemia en el desarrollo infantil: consecuencias a largo plazo. Rev perú med exp salud publica. 2017;34(4):716-722. DOI:https://doi.org/10.17843/rpmesp.2017.344.3346

23. Guzmán MJ, Guzmán Z JL, Llanos de los ReyesGarcía M.J. Significado de la anemia en las diferentes etapas de la vida. Enferm glob. 2016;15(43): 407418. (Citado 1 de noviembre 2017) Disponible en: http://scielo.isciii.es/scielo.php?script=sci_arttext\&p 
$\mathrm{id}=\mathrm{S} 169561412016000300015 \& \operatorname{lng}=\mathrm{es}$.

24. Laca F. Actitudes y comportamientos en las situaciones de conflicto. Enseñanza e Investigación en Psicología. 2005; 10(1): 117-126.

25. Vargas J. Actitudes de las madres de niños de 6 a 36 meses hacia la suplementación con multimicronutrientes en un establecimiento de salud de Lima. Tesis de Grado. Lima, Perú: Universidad Nacional Mayor de San Marcos; 2017.

26. Junco J. Identificación de los factores que contribuyen y limitan la efectividad del programa de suplementación con multimicronutrientes en la reducción de la anemia de niños menores de tres años del ámbito rural de Vinchos de Ayacucho. Tesis de Grado. Perú: Pontificia Universidad Católica del Perú; 2015.

27. Huamán L, Aparco J, Núñez E, Gonzáles J, Mayta P. Consumo de suplementos con multimicronutrientes Chispitas y anemia en niños de 6 a 35 meses: estudio transversal en el contexto de una intervención poblacional en Apurímac, Perú. Rev Perú med exp salud publica. 2012 ; 29 (3): 314-323. DOI: https:// doi.org/10.17843/rpmesp.2012.293.363

28. Kodish S, Rah JH, Kraemer K, de Pee S, Gittelsohn J. Estudio sobre el bajo uso de micronutrientes en polvo en el campo de refugiados de Kakuma en Kenia: resultados de un estudio cualitativo. Food Nutr Bull. 2011;32(3):292-303.
29. Ip H, Hyder SM, Haseen F, Rahman M, Zlotkin SH. Mejora de la adherencia y tasas de curación de la anemia con la administración flexible de micronutrientes Sprinkles: un nuevo enfoque de salud pública para el control de la anemia. Eur J Clin Nutr. 2009; 63(2): 165-72.

30. Hinostroza M. Barreras y motivaciones en el nivel de adherencia a la suplementación con multimicronutrientes en madres de niños menores de 36 meses, cercado de Lima. Tesis de Grado. Lima, Perú: Universidad Nacional Mayor De San Marcos; 2015.

31. Gutiérrez M. Efecto de las prácticas de la suplementación con multimicronutrientes y consumo de hierro dietético en los niveles de hemoglobina en niños con anemia de 6 a 36 meses de edad del centro de salud metropolitano. Tesis de Grado. Puno: Universidad Nacional del Altiplano; 2016.

32. Organización Mundial de la Salud; Organización Panamericana de la Salud. Adherencia a los tratamientos a largo plazo: pruebas para la acción. Washington D.C: OMS; 2014.

Recibido: $\quad 01 / 06 / 2019$

Aceptado: 15/11/2019 